\title{
Singlet-doublet Dirac dark matter and neutrino masses
}

\author{
Diego Restrepo๑* and Andrés Rivera $\odot^{\dagger}$ \\ Instituto de Física, Universidad de Antioquia, Calle 70 No. 52-21, Apartado Aéreo 1226, Medellín, Colombia \\ Walter Tangarife $\odot^{*}$ \\ Department of Physics, Loyola University Chicago, \\ 1032 West Sheridan Road, Chicago, Illinois 60660, USA
}

(Received 3 July 2019; published 26 August 2019)

\begin{abstract}
We examine an extension of the Standard Model that addresses the dark matter puzzle and generates Dirac neutrino masses through the radiative seesaw mechanism. The new field content includes a scalar field that plays an important role in setting the relic abundance of dark matter. We analyze the phenomenology in the light of direct, indirect, and collider searches of dark matter. In this framework, the dark matter candidate is a Dirac particle that is a mixture of new singlet-doublet fields with mass $m_{\chi_{1}^{0}} \lesssim 1.1 \mathrm{TeV}$. We find that the allowed parameter space of this model is broader than the well-known Majorana dark matter scenario.
\end{abstract}

DOI: 10.1103/PhysRevD.100.035029

\section{INTRODUCTION}

There is substantial evidence that supports the existence of dark matter (DM). Some of that evidence includes velocity dispersion in clusters of galaxies [1] (see [2] for a recent review), galaxy rotation curves [3,4], the cosmic microwave background (CMB) [5], galaxy cluster collisions [6], and weak and strong gravitational lensing $[7,8]$. Currently, it is well established that DM makes up about $27 \%$ of the energy density of the Universe, although its nature and properties remain an open puzzle. $N$-body simulations of early structure formation and CMB data suggest that DM is made up of cold, collisionless particles [9]. In light of this indication, there has been a vast exploration of candidates for DM during the last few decades, but no detection experiment has been able to find the DM particle. In addition to the DM problem, one of the open issues in the Standard Model (SM) is the fact that neutrinos have mass, which has been confirmed by neutrinooscillation experiments [10]. The DM problem and the neutrino mass puzzle make clear the necessity of beyondthe-Standard-Model physics.

In this article, we study these two puzzles within a simple extension of the singlet-doublet Dirac dark matter $\left(\mathrm{SD}^{3} \mathrm{M}\right)$

\footnotetext{
*restrepo@udea.edu.co

†afelipe.rivera@udea.edu.co

¥wtangarife@luc.edu
}

Published by the American Physical Society under the terms of the Creative Commons Attribution 4.0 International license. Further distribution of this work must maintain attribution to the author(s) and the published article's title, journal citation, and DOI. Funded by SCOAP ${ }^{3}$. model [11]. In singlet-doublet DM scenarios, a singlet and a doublet fermionic field are added to the SM and a mixture of such fields is a Majorana DM candidate [12-23]. In the singlet-doublet Dirac dark matter model, the DM candidate is a Dirac particle, which opens a vector portal to the SM via the $Z$ gauge boson, resulting in a richer phenomenology. In general, this portal is not present in the singletdoublet DM model with Majorana fermions, which is a generalization of the supersymmetric Higgsino-bino case [19]. The $\mathrm{SD}^{3} \mathrm{M}$ model addresses the DM problem while being consistent with indirect and direct experiments, as studied in Ref. [11]. In addition, it can be tested in future experiments such as LZ [24] and its low mass region could be probed at the Large Hadron Collider (LHC). This simple model, however, does not generate neutrino masses. Thus, in this work, we enlarge this framework with a minimal set of scalar singlet fields in order to explain Dirac masses of SM neutrinos. These Dirac neutrino masses are generated at one-loop level in a similar fashion as in the scotogenic class of models introduced first in [25]. An additional feature of this mechanism is the enhancement of the scalar portal that is suppressed in the minimal framework of the $\mathrm{SD}^{3} \mathrm{M}$ model studied in Ref. [11].

We describe our model in Sec. II. In Sec. III, we present the generation of the neutrino masses. Section IV includes the DM analysis and numerical results, and we close with Conclusions.

\section{DESCRIPTION OF THE MODEL}

In this model, we extend the symmetry of the SM with two discrete symmetries, $\mathbb{Z}_{2}$ and $\mathbb{Z}_{2}^{\prime}$. $\mathbb{Z}_{2}$ stabilizes the DM 
TABLE I. Particle content of the model.

\begin{tabular}{lcccc}
\hline \hline $\begin{array}{l}\text { Leptons and } \\
\text { scalars fields }\end{array}$ & $\left(\mathrm{SU}(2)_{L}, \mathrm{U}(1)_{Y}\right)$ & $\mathbb{Z}_{2}(\mathrm{DM})$ & $\mathbb{Z}_{2}^{\prime}$ & $U(1)_{B-L}$ \\
\hline$L_{\beta}=\left(\begin{array}{c}\nu_{L} \\
l_{L}\end{array}\right)_{\beta}$ & $(2,-1 / 2)$ & + & + & -1 \\
$l_{R}^{\alpha}$ & $(1,0)$ & + & + & -1 \\
$H=\left(H^{+}, \frac{h^{0}+v}{\sqrt{2}}\right)^{T}$ & $(2,1 / 2)$ & + & + & 0 \\
$S$ & $(1,0)$ & + & - & 0 \\
$\sigma_{i}$ & $(1,0)$ & - & - & 0 \\
$\psi_{L}$ & $(1,0)$ & - & + & -1 \\
$\psi_{R}$ & $(1,0)$ & - & - & -1 \\
$\Psi=\left(\begin{array}{c}\Psi^{0} \\
\Psi^{-}\end{array}\right)$ & $(2,-1 / 2)$ & - & - & -1 \\
$\nu_{R}^{\alpha}$ & $(1,0)$ & + & - & -1 \\
\hline \hline \multicolumn{7}{c}{}
\end{tabular}

particle and $\mathbb{Z}_{2}^{\prime}$ forbids the generation of neutrino masses via the seesaw mechanism at tree level [26,27]. All SM particles are even under these discrete symmetries. This model also includes the following additional fields: a real scalar singlet $S=\left(S^{0}+v_{S}\right) / \sqrt{2}$, two real scalar singlets $\sigma_{i}$ (which are needed to obtain a rank-2 neutrino mass matrix), two chiral fermionic singlets $\psi_{L}$ and $\psi_{R}$, one Dirac SU(2) vectorlike fermion $\Psi$ with hypercharge $-1 / 2$, and three right-handed neutrinos $\nu_{R}^{\alpha}$. In addition, we assume that global $U(1)_{B-L}$ is conserved and that the new fermions are charged under this symmetry. A result of this assumption is that Majorana mass terms are forbidden, leading to Dirac neutrino masses. The particle content is also listed in Table I. ${ }^{1}$

The most general Lagrangian, invariant under the symmetries mentioned above, contains the terms

$$
\begin{aligned}
\mathcal{L} \supset & -M_{\Psi} \bar{\Psi} \Psi-V\left(H, \sigma_{i}, S\right) \\
& +\left[h_{a}^{\beta i} \bar{L}_{\beta} \Psi \sigma_{i}+h_{b}^{\alpha i} \overline{\psi_{L}} \nu_{R \alpha} \sigma_{i}+h_{c} \overline{\psi_{R}} \psi_{L} S\right. \\
& \left.+h_{d} \bar{\Psi} \tilde{H} \psi_{R}+\text { H.c. }\right],
\end{aligned}
$$

where $h$ 's are Yukawa couplings, which we assume to be real parameters for the sake of simplicity, and $\tilde{H}=i \sigma_{2} H^{*}$. Notice that the vectorlike fermion $\Psi$ can be written in terms of two chiral doublets $\Psi_{L}=\left(\Psi_{L}^{0}, \Psi_{L}^{-}\right)^{T}$ and $\widetilde{\left(\Psi_{R}\right)}=\left(-\left(\Psi_{R}^{-}\right)^{\dagger},\left(\Psi_{R}^{0}\right)^{\dagger}\right)^{T}$ with opposite hypercharge [28], as shown in the Appendix.

\footnotetext{
${ }^{1}$ A different $U(1)_{B-L}$ charge assignment, in radiative Dirac neutrino mass models, was made in Ref. [28] for the case of complex $\sigma_{i}$.
}

The scalar potential is given by

$$
\begin{aligned}
V\left(H, \sigma_{i}, S\right)= & -\mu^{2} H^{\dagger} H+\frac{\lambda_{1}}{2}\left(H^{\dagger} H\right)^{2}+\frac{1}{2} m_{\sigma_{i}}^{2} \sigma_{i}^{2} \\
& +\lambda_{i}^{\sigma H} H^{\dagger} H \sigma_{i}^{2}+\frac{\lambda_{i}^{\sigma}}{2} \sigma_{i}^{4}+\frac{1}{2} m_{S}^{2} S^{2} \\
& +\lambda_{S H} H^{\dagger} H S^{2}+\lambda^{S \sigma_{i}} S^{2} \sigma_{i}^{2}+\frac{\lambda^{S}}{2} S^{4} .
\end{aligned}
$$

The condition that the potential is bounded from below is fulfilled by imposing $\mu^{2}>0, m_{\sigma i}^{2}>0, m_{S}^{2}>0$, together with the copositivity of the potential [29], which yields

$$
\lambda_{1} \geq 0, \quad \lambda_{i}^{\sigma} \geq 0, \quad \lambda^{S} \geq 0,
$$

$\frac{\lambda_{i}^{\sigma H}}{2}+\sqrt{\lambda_{1} \lambda_{i}^{\sigma}} \geq 0, \quad \frac{\lambda_{S H}}{2}+\sqrt{\lambda_{1} \lambda^{S}} \geq 0, \quad \frac{\lambda^{S \sigma}}{2}+\sqrt{\lambda_{i}^{\sigma} \lambda^{S}} \geq 0$,

$$
\begin{aligned}
& \sqrt{\lambda_{1} \lambda_{i}^{\sigma} \lambda^{S}}+\frac{\lambda_{i}^{\sigma H}}{2} \sqrt{\lambda^{S}}+\frac{\lambda_{S H}}{2} \sqrt{\lambda_{i}^{\sigma}}+\frac{\lambda_{i}^{S \sigma_{i}}}{2} \sqrt{\lambda_{1}} \\
& +\sqrt{2\left(\frac{\lambda_{i}^{\sigma H}}{2}+\sqrt{\lambda_{1} \lambda_{i}^{\sigma}}\right)\left(\frac{\lambda_{S H}}{2}+\sqrt{\lambda_{1} \lambda^{S}}\right)\left(\frac{\lambda^{S \sigma_{i}}}{2}+\sqrt{\lambda_{i}^{\sigma} \lambda^{S}}\right)} \geq 0 .
\end{aligned}
$$

These conditions are trivially satisfied if we demand that all $\lambda$ 's be positive.

\section{A. Symmetry breaking and spectrum}

The scalar potential (2) allows a vacuum expectation value (VEV) for the singlet scalar, $\langle S\rangle=v_{S} / \sqrt{2}$, in addition to the Higgs VEV, $\langle H\rangle=v / \sqrt{2}$. These VEVs are given by the tadpole equations

$$
\begin{aligned}
& t_{H}=\left(\frac{\partial V}{\partial v}\right)=-\mu^{2} v+\frac{\lambda_{1} v^{3}}{2}+\frac{\lambda_{S H}}{2} v v_{S}^{2}=0, \\
& t_{S}=\left(\frac{\partial V}{\partial v_{S}}\right)=m_{S}^{2} v_{S}+\lambda_{S H} v^{2} v_{S}+\lambda^{S} v_{S}^{3}=0,
\end{aligned}
$$

which are used to eliminate the parameters $\mu$ and $m_{S}$. The scalar spectrum contains the $\mathbb{Z}_{2}$-even scalars $h^{0}, S^{0}$, and $\mathbb{Z}_{2}$-odd scalars $\sigma_{i}$. In the basis $\left(h^{0}, S^{0}\right)$, the mass matrix for the $\mathbb{Z}_{2}$-even scalars is given by

$m_{h}^{2}=\left(\begin{array}{cc}-\mu^{2}+\frac{1}{2} v_{S}^{2} \lambda_{S H}+\frac{3 \lambda_{1} v^{2}}{2} & v v_{S} \lambda_{S H} \\ v v_{S} \lambda_{S H} & \frac{m_{s}^{2}}{2}+\frac{3}{2} \lambda^{S} v_{S}^{2}+\frac{v^{2} \lambda_{S H}}{2}\end{array}\right)$,

which is diagonalized by a unitary transformation 


$$
Z^{H} m_{h}^{2} Z^{H \dagger}=m_{h, \text { diag }}^{2},
$$

such that

$$
\left(\begin{array}{l}
h_{0} \\
S^{0}
\end{array}\right)=Z^{H}\left(\begin{array}{l}
h_{1} \\
h_{2}
\end{array}\right)=\left(\begin{array}{cc}
\cos \alpha & \sin \alpha \\
-\sin \alpha & \cos \alpha
\end{array}\right)\left(\begin{array}{l}
h_{1} \\
h_{2}
\end{array}\right) .
$$

The lightest eigenstate, $h_{1}$, is identified with the SM Higgs boson, whereas the heavier one will be a heavy Higgs boson not yet discovered at the LHC. The existence of a second Higgs can be beneficial in order to stabilize the metastable electroweak vacuum of the SM, as argued in Ref. [30]. However, some constraints need to be taken into account. The Higgs-boson mixing (10) generates the effective interaction terms

$$
\begin{aligned}
\mathcal{L} \supset & \frac{h_{1} \cos \alpha+h_{2} \sin \alpha}{v} \\
& \times\left(2 m_{W}^{2} W_{\mu}^{+} W^{\mu-}+m_{Z}^{2} Z_{\mu} Z^{\mu}-\sum_{f} m_{f} \bar{f} f\right),
\end{aligned}
$$

which suppress the partial decay of $h_{1}$ to SM fields by the factor $\sim \cos ^{2} \alpha$. Similarly, the heavier scalar $h_{2}$ could have a decay width $\Gamma\left(h_{2} \rightarrow h_{1} h_{1}\right) \sim \sin ^{2} \alpha$ if it is kinematically allowed. In addition, $h_{2}$ is constrained by the electroweak oblique parameters since, for $m_{h_{2}} \gg m_{h_{1}}$, it has been shown that [30]

$$
\begin{gathered}
T \approx-\frac{3}{8 \pi \cos ^{2} \theta_{W}} \sin ^{2} \alpha \log \left(m_{h_{2}} / 211 \mathrm{GeV}\right), \\
S \approx \frac{1}{6 \pi} \sin ^{2} \alpha \log \left(m_{h_{2}} / 81 \mathrm{GeV}\right) .
\end{gathered}
$$

Further constraints are provided by LEP and LHC searches for Higgs-like scalars. For instance, processes such as $h_{i} \rightarrow \gamma \gamma, h_{2} \rightarrow Z Z, h_{2} \rightarrow W W$, etc., have been analyzed in the literature [31-34]. As shown in Ref. [30], by combining the experimental constraints and taking care of the vacuum stability in the evolution of the renormalization group equations up to the Planck scale, these observables and constraints are under control if we demand a mixing $|\sin \alpha| \lesssim 0.3$, which has been taken into account in this work. On the other hand, the $\mathbb{Z}_{2}$-odd scalar sector is assumed to be already in the diagonal basis,

$$
m_{\sigma}^{2}=\left(\begin{array}{cc}
m_{\sigma 1}^{2}+v^{2} \lambda_{1}^{\sigma_{1} H}+v_{S}^{2} \lambda^{S \sigma_{1}} & 0 \\
0 & m_{\sigma 2}^{2}+v^{2} \lambda_{2}^{\sigma_{2} H}+v_{S}^{2} \lambda^{S \sigma_{2}}
\end{array}\right) .
$$

While the lightest of these scalars could be a suitable candidate for DM, in this work we focus instead on fermionic DM. The scalar DM phenomenology is expected to be rather similar to the one in the Majorana version for both DM and neutrino masses [19,35]. Therefore, we will assume the $\sigma_{i}$ fields to be heavy $\left(m_{\sigma_{i}}>1 \mathrm{TeV}\right)$ while playing an important role only in the generation of neutrino masses, as shown in the next section.

Regarding the $\mathbb{Z}_{2}$-odd fermionic sector, this model contains one charged Dirac fermion $\Psi^{ \pm}$with mass $M_{\Psi}$ and two neutral Dirac fermions, $\chi_{j}^{0}(j=1,2)$. In the basis $N_{L i}=\left(\Psi_{L}^{0}, \psi_{L}\right), \quad N_{R i}^{\dagger}=\left(\left(\Psi_{R}^{0}\right)^{\dagger},\left(\psi_{R}\right)^{\dagger}\right)$, the fermionic mass matrix is given by

$$
m_{\psi^{0}}=\left(\begin{array}{cc}
M_{\Psi} & \frac{h_{d} v}{\sqrt{2}} \\
0 & M_{N}
\end{array}\right)
$$

where $M_{N}=h_{c} v_{S} / \sqrt{2}$ is the Dirac mass term for $\psi_{L, R}$, which results after the $\mathbb{Z}_{2}^{\prime}$ symmetry breaking. This matrix is diagonalized by the biunitary transformation

$$
V^{*} m_{\psi^{0}} U^{\dagger}=m_{\chi_{i}^{0}}^{\mathrm{diag}},
$$

where the mass eigenstates, $\chi_{j}^{0}=\left(\chi_{L}, \chi_{R}^{\dagger}\right)_{j}$, are defined by

$$
\begin{aligned}
& \chi_{L j}=V_{j i} N_{L i}=\left(\begin{array}{cc}
\cos \theta_{L} & \sin \theta_{L} \\
-\sin \theta_{L} & \cos \theta_{L}
\end{array}\right)\left(\begin{array}{c}
\Psi_{L}^{0} \\
\psi_{L}
\end{array}\right), \\
& \chi_{R j}^{\dagger}=U_{j i} N_{R i}^{\dagger}=\left(\begin{array}{cc}
\cos \theta_{R} & \sin \theta_{R} \\
-\sin \theta_{R} & \cos \theta_{R}
\end{array}\right)\left(\begin{array}{l}
\left(\Psi_{R}^{0}\right)^{\dagger} \\
\left(\psi_{R}\right)^{\dagger}
\end{array}\right),
\end{aligned}
$$

where $\theta_{L, R}$ are mixing angles. In this work, the lightest of these Dirac fermions, $\chi_{1}^{0}$, is the candidate for the DM particle. Notice our choice to parametrize the fermionic sector using $m_{\chi_{1}^{0}}, m_{\chi_{2}^{0}}, \theta_{L}$, and $\theta_{R}$, instead of $M_{\Psi}, h_{c}, h_{d}$, and $v_{S}$.

\section{DIRAC NEUTRINO MASSES}

In this framework, the scalars $H$ and $S$ acquire VEVs. As a result of this symmetry breaking, neutrinos get masses via the five-dimensional effective operator

$$
\mathcal{L}_{5}^{D}=-\frac{g_{\alpha \beta}}{\Lambda} \bar{L}_{\alpha} \tilde{H} \nu_{R \beta} S+\text { H.c. }
$$

which is generated at the one-loop level. The authors of Ref. [36] have performed a systematic study of the oneloop topologies that give rise to this operator. ${ }^{2}$ In our specific scenario, Dirac neutrino masses arise from the oneloop diagram shown in Fig. 1. In the limit of low neutrino momentum, that diagram yields the mass matrix

\footnotetext{
${ }^{2}$ In particular, the model proposed in this work is similar to the topology T1-2-A-I ( $\alpha=0)$ in Ref. [36]. However, in that case all new fermions are vectorlike. Instead, we use chiral fermions with fewer degrees of freedom.
} 


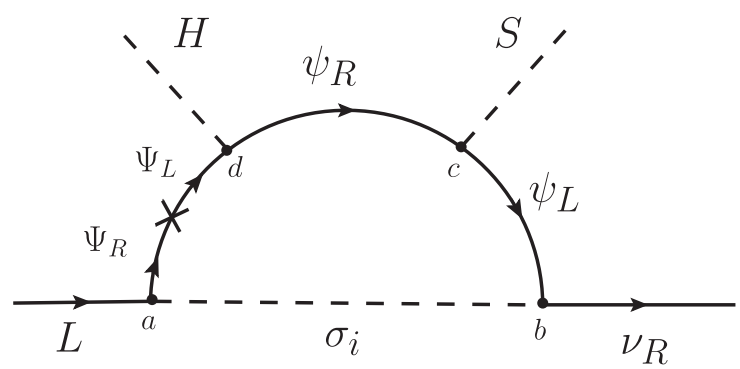

FIG. 1. One-loop generation of Dirac neutrino masses. The arrows represent the flow of $U(1)_{B-L}$ charges.

$$
\begin{aligned}
\mathcal{M}_{\alpha \beta}= & \sum_{i=1}^{2} \sum_{j=1}^{2} \frac{U_{j 1} V_{j 2}}{16 \pi^{2}} \times h_{b}^{\alpha i} h_{a}^{\beta i} m_{\chi_{j}^{0}} \\
& \times\left[\frac{m_{\chi_{j}^{0}}^{2} \ln \left(m_{\chi_{j}^{0}}^{2}\right)-m_{\sigma_{i}}^{2} \ln \left(m_{\sigma_{i}}^{2}\right)}{\left(m_{\chi_{j}^{0}}^{2}-m_{\sigma_{i}}^{2}\right)}\right], \\
= & \sum_{i=1}^{2} h_{b}^{\alpha i} \times \Lambda_{i} \times h_{a}^{\beta i},
\end{aligned}
$$

where $\Lambda_{i}$ is the loop factor, defined as

$$
\begin{aligned}
\Lambda_{i} & =\sum_{j=1}^{2} \frac{U_{j 1} V_{j 2}}{16 \pi^{2}} \times m_{\chi_{j}^{0}} \times\left[\frac{m_{\chi_{j}^{0}}^{2} \ln \left(m_{\chi_{j}^{0}}^{2}\right)-m_{\sigma_{i}}^{2} \ln \left(m_{\sigma_{i}}^{2}\right)}{\left(m_{\chi_{j}^{0}}^{2}-m_{\sigma_{i}}^{2}\right)}\right], \\
& =\sum_{j=1}^{2} \frac{U_{j 1} V_{j 2}}{16 \pi^{2}} \times m_{\chi_{j}^{0}} \times\left[\frac{m_{\chi_{j}^{0}}^{2}}{\left(m_{\chi_{j}^{0}}^{2}-m_{\sigma_{i}}^{2}\right)} \ln \left(\frac{m_{\chi_{j}^{0}}^{2}}{m_{\sigma_{i}}^{2}}\right)\right] .
\end{aligned}
$$

In the last equation, we used the relation

$$
\sum_{j}^{2} m_{\chi_{j}^{0}} U_{j 1} V_{j 2}=0
$$

which is a consequence of Eqs. (15) and (16).

We need to set the correct Yukawa couplings in the Lagrangian (1) in order to reproduce the current neutrino oscillation data to $3 \sigma$ [10]. That is, we need to invert the problem and use the neutrino parameters to choose our Yukawa couplings. This can be done by using the fact that, in the basis where $\nu_{R}^{\alpha}$ are mass eigenstates, the neutrino mass matrix can be written as [37]

$$
\mathcal{M}_{\alpha \beta}=\left(U_{\mathrm{PMNS}}\right)_{\alpha \beta}\left(m_{\nu}\right)_{\beta},
$$

where $U_{\text {PMNS }}$ is the Pontecorvo-Maki-Nakagawa-Sakata matrix [38] and $m_{\nu}$ are the neutrino mass eigenvalues. It is well known that current neutrino oscillation data allow for normal or inverted ordering, $m_{\nu 1}<m_{\nu 2}<m_{\nu 3}$ or $m_{\nu 3}<m_{\nu 1}<m_{\nu 2}$, respectively. In this work, we choose the normal ordering. Using the Eqs. (19) and (22), we obtain 12 unknown parameters, $h_{a}^{\beta i}, h_{b}^{\alpha i}$, with 9 equations.
We can further simplify our analysis by imposing $m_{\nu 1}=0$, which allows us to set $h_{a}^{1 i}=0$, leaving the couplings $h_{a}^{2 i}$ and $h_{a}^{3 i}$ as free parameters. With these assumptions, we obtain the following relations:

$$
\begin{aligned}
h_{a}^{1 i} & =0, \\
h_{a}^{2 i, 3 i} & =\text { free, } \\
h_{b}^{\alpha 1} & =-\frac{1}{\Lambda_{1}}\left(\frac{h_{a}^{32} m_{\nu 2} U_{\alpha 2}-h_{a}^{22} m_{\nu 3} U_{\alpha 3}}{h_{a}^{22} h_{a}^{31}-h_{a}^{21} h_{a}^{32}}\right), \\
h_{b}^{\alpha 2} & =-\frac{1}{\Lambda_{2}}\left(\frac{h_{a}^{31} m_{\nu 2} U_{\alpha 2}-h_{a}^{21} m_{\nu 3} U_{\alpha 3}}{h_{a}^{22} h_{a}^{31}-h_{a}^{21} h_{a}^{32}}\right) .
\end{aligned}
$$

It is noteworthy that, with this choice of parameters, some lepton-flavor-violation (LFV) processes such as $\mu \rightarrow e \gamma$ are suppressed since they are proportional to the $h_{a}^{1 i}$ coupling. However, other processes, like $\tau \rightarrow \mu \gamma$, are still allowed with much lower experimental restrictions.

\section{DARK MATTER}

In this work, the Dirac fermion $\chi_{1}^{0}$ is the DM candidate while the scalars $\sigma_{i}$ are chosen to be much heavier than $\chi_{j}^{0}$. In this section, we discuss the main process that sets the relic abundance of DM as well as the direct detection of such a particle.

\section{A. Dark matter relic density}

In the class of models that we study in this article, $\chi_{1}^{0}$ couples to the Higgs and to the $Z$ boson through the singlet-doublet mixing. This implies that the couplings of the DM particle to the $Z$ vector are largely constrained by direct detection experiments, leading to a mostly singlet DM candidate as seen numerically in the next section. In Ref. [11], this fact restricted the allowed parameter space to quasidegenerate mass eigenstates for the fermionic fields and the DM abundance was determined mainly through coannihilations. In our work, the presence of the additional scalar $S$ adds new annihilation channels, opening up the range of masses for the fermions and providing a richer phenomenology. Specifically, the processes involved in the calculation of the DM relic abundance include $\chi_{i}^{0} \bar{\chi}_{j}^{0} \rightarrow h_{k} h_{l}, \chi_{i}^{0} \bar{\chi}_{j}^{0} \rightarrow W^{+} W^{-}, \chi_{i}^{0} \bar{\chi}_{j}^{0} \rightarrow$ $Z Z, \quad \chi^{ \pm} \chi^{\mp} \rightarrow f \bar{f}, \quad \chi_{i}^{0} \chi^{+} \rightarrow f \bar{f}^{\prime}, \quad \chi_{i}^{0} \chi^{ \pm} \rightarrow A / Z W^{ \pm}, \quad$ and $\chi^{ \pm} \chi^{\mp} \rightarrow W^{+} W^{-}$. As explained in the next section, our numerical analysis takes into account all these channels; however, the most relevant process is $\chi_{1}^{0} \bar{\chi}_{1}^{0} \rightarrow h_{2} h_{2}$, which gets contributions from the diagrams shown in Fig. 2.

The early thermal evolution of our DM candidate follows the standard weakly interacting massive particle (WIMP) freeze-out mechanism. In the initial state, the DM species was in thermal equilibrium with the rest of the particles in the Universe. As the Universe adiabatically cools down to a temperature below the DM mass, the DM annihilation rate 


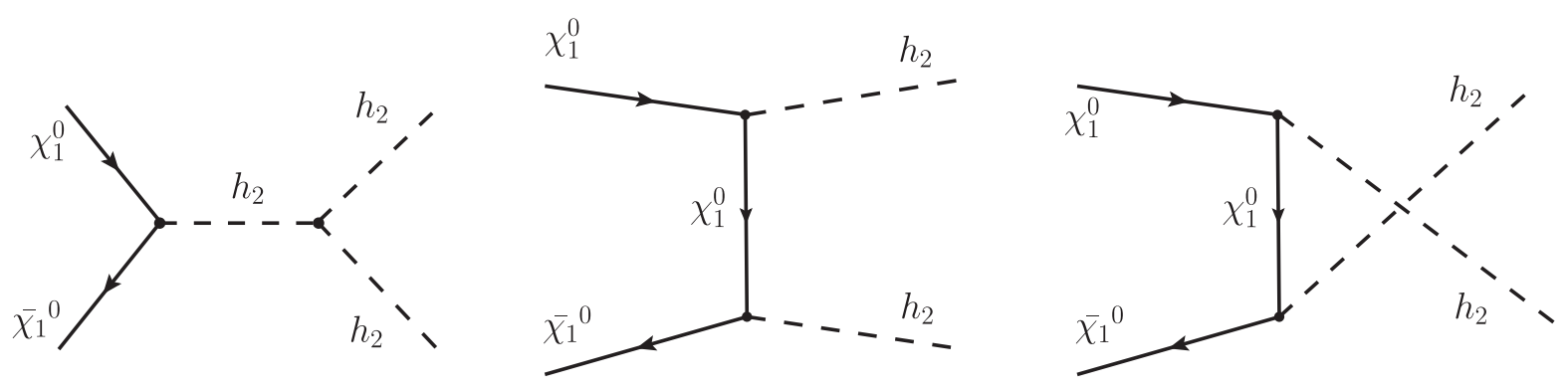

FIG. 2. Diagrams that contribute to the annihilation process $\chi_{1}^{0} \bar{\chi}_{1}^{0} \rightarrow h_{2} h_{2}$.

is overtaken by the expansion of the Universe, $\Gamma \ll H$, and a relic density of DM is frozen out. The current relic abundance of DM is computed by solving the Boltzmann equation, which yields [39]

$$
\Omega_{\chi}=2 \sqrt{\frac{4 \pi^{3} g_{*}\left(m_{\chi}\right)}{45}} \frac{8 \pi}{90 H_{0}^{2}} \frac{x_{f}}{\langle\sigma v\rangle} \frac{T_{0}^{3}}{M_{\mathrm{Pl}}^{3}},
$$

where $\langle\sigma v\rangle$ is the thermally averaged annihilation cross section, $g_{*}(m)$ is the effective number of degrees of freedom at $T=m$, and $x_{f} \equiv m / T_{\text {freeze-out }}$. The factor of 2 in front of the right-hand side of the equation above is due to the fact that we have a Dirac particle and $n_{\mathrm{DM}}=n_{\chi}+n_{\bar{\chi}}$ [40]. The partial-wave expansion of the annihilation cross section, $\langle\sigma v\rangle \approx a+b v^{2}+\mathcal{O}\left(v^{4}\right)$, leads to the well-known expression

$$
\Omega_{\chi} h^{2} \approx 2 \frac{1.04 \times 10^{9} x_{f}}{M_{\mathrm{Pl}} \sqrt{g_{*}\left(m_{\chi}\right)}\left(a+3 b / x_{f}\right)},
$$

where $h$ is today's Hubble parameter in units of $100 \mathrm{~km} / \mathrm{s} /$ Mpc. The $\chi_{1}^{0} \bar{\chi}_{1}^{0} \rightarrow h_{2} h_{2}$ annihilation cross section has no $s$-wave contribution, which means that $a=0$. In order to achieve the measured relic density, $\Omega_{\chi} h^{2}=$ $0.1200 \pm 0.0012$ [5], the annihilation cross section is required to be approximately $\langle\sigma v\rangle \sim 3 \times 10^{-26} \mathrm{~cm}^{3} \mathrm{~s}^{-1}$. For illustrative purposes, let us write the specific expression for the cross section in the limit where DM is purely singlet, $\chi_{j}^{0}=\left(\begin{array}{c}\psi_{L} \\ \psi_{R}^{\dagger}\end{array}\right)$ :

$$
\begin{aligned}
\langle\sigma v\rangle= & \frac{h_{c}^{2} \sqrt{1-\mu^{2}}}{16 \pi m_{\chi_{0}}^{2}}\left(\frac{9\left(\lambda_{S H}\right)^{2} v_{S}^{2}}{16 m_{\chi_{0}}^{2}\left(\mu^{2}-4\right)^{2}}\right. \\
& \left.-\frac{h_{c} \lambda_{S H} v_{S}\left(20-13 \mu^{2}+2 \mu^{4}\right)}{2 \sqrt{2} m_{\chi_{0}}\left(\mu^{2}-4\right)^{2}\left(\mu^{2}-2\right)^{2}}+\frac{h_{c}^{2}\left(9-8 \mu^{2}+2 \mu^{4}\right)}{6\left(\mu^{2}-2\right)^{2}}\right) v^{2}, \\
& =b v^{2}
\end{aligned}
$$

where $\mu \equiv m_{\chi_{0}} / m_{h_{2}}<1$. The first term corresponds to the $s$ channel while the last one comes from the $t$ and $u$ channels and their interference. The second term results from the interference between the $s$ and the $t, u$ channels (see Fig. 2). In the next section, we present the numerical results of this computation and the corresponding relic abundance. Finally, let us mention that there is a clear consequence of having $p$-wave annihilation of DM for indirect-detection searches. Since $\sigma v \propto v^{2}$, the annihilation rate is suppressed by several orders of magnitude in the low-velocity limit (today) compared to the value in the early Universe, escaping the bounds from current indirect searches, which require $\sigma v \lesssim 3 \times 10^{-26} \mathrm{~cm}^{3} \mathrm{~s}^{-1}$.

\section{B. Direct detection of DM}

As mentioned above, since $\chi_{1}^{0}$ couples to scalars and the $Z$ boson, there are direct and indirect detection restrictions that can be imposed on this model. Regarding elastic scattering of $\chi_{1}^{0}$ with nuclei, we have two different contributions, the scalar/vector or spin-independent (SI) interaction and the axial-vector or spin-dependent (SD) interaction. It is noteworthy that in the SUSY analog of the singlet-doublet model, i.e., the Higgsino-bino model, the SI interaction is only due to the scalar portal. In that case, the vector portal with the $Z$ boson is closed since the DM particles are Majorana fermions. However, in our scenario, the SI interaction of DM with nucleons contains both portals: a $t$ channel mediated by the Higgs bosons $h_{k}$ and a $t$ channel mediated by the $Z$ gauge boson, which correspond to the diagrams shown in Fig. 3. We use the standard nucleon-form-factor formalism to incorporate these processes into the WIMP-nucleon amplitudes [41]. Given the interaction Lagrangian $\mathcal{L}_{e, o}^{\mathrm{SI}}=$ $\lambda_{N, e} \bar{\psi}_{\chi} \psi_{\chi} \psi_{N} \psi_{N}+\lambda_{N, o} \bar{\psi}_{\chi} \gamma^{\mu} \psi_{\chi} \psi_{N} \gamma_{\mu} \psi_{N}, N=p, n$, the scattering cross section per nucleus is given by
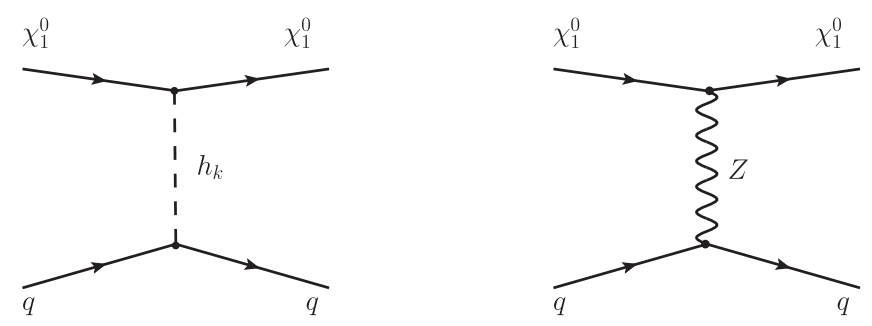

FIG. 3. SI-independent DM-nucleon interactions: scalar (left) and vector (right) portals. 


$$
\sigma_{0}^{\mathrm{SI}}=\frac{4 \mu_{\chi}^{2}}{\pi}\left(\lambda_{p} Z+\lambda_{n}(A-Z)\right)^{2},
$$

where $\mu_{\chi}=m_{\chi} M_{A} /\left(m_{\chi}+M_{A}\right)$ is the WIMP-nucleus reduced mass; $Z$ is the nucleus charge; $A$ is the total number of nucleons; and $\lambda_{p}, \lambda_{n}$ are related to $\lambda_{N, e}, \lambda_{N, o}$, as we will show in the next paragraph. When implementing experimental bounds, the relevant quantity is the scattering cross section per nucleon, which is written as

$$
\sigma_{N}^{\mathrm{SI}}=\frac{m_{N}^{2}}{\mu_{\chi}^{2} A^{2}} \sigma_{0}^{\mathrm{SI}}
$$

where $m_{N}$ is the nucleon mass.

Using the nucleon-quark operator formalism, $\lambda_{p}, \lambda_{n}$ are found to be [41]

$\lambda_{p}=\frac{\lambda_{p, e} \pm \lambda_{p, o}}{2}=\left(g_{\chi_{1}^{0} \chi_{1}^{0} h_{k}} \frac{1}{m_{h_{k}}^{2}} \frac{m_{p} \sum_{q} f_{q}^{p}}{v} \pm \sum_{q=u, d} f_{V q}^{p} \lambda_{q, o}\right) / 2$

$\lambda_{n}=\frac{\lambda_{n, e} \pm \lambda_{n, o}}{2}=\left(g_{\chi_{1}^{0} \chi_{1}^{0} h_{k}} \frac{1}{m_{h_{k}}^{2}} \frac{m_{n} \sum_{q} f_{q}^{n}}{v} \pm \sum_{q=u, d} f_{V q}^{n} \lambda_{q, o}\right) / 2$

where the $+(-)$ signs correspond to WIMP (anti-WIMP) interaction; $\sum_{q} f_{q}^{p} \approx \sum_{q} f_{q}^{n}=f_{N} \approx 0.3$ is the form factor for the scalar interaction $[42,43] ; f_{V q}^{N}$ counts the number of quarks $u, d$ inside the nucleon $\left(f_{V u}^{p}=2, f_{V d}^{p}=1, f_{V u}^{n}=1\right.$, $f_{V d}^{n}=2$ ); and $\lambda_{q, o}$ are the vector form factors, which, in our model, follow the relations

$$
\begin{aligned}
\sum_{q=u, d} f_{V q}^{p} \lambda_{q, o}= & \frac{M_{Z}\left(\cos ^{2} \theta_{L}+\cos \theta_{R}^{2}\right)}{2 v} \times \frac{1}{M_{Z}^{2}} \\
& \times \frac{e}{4 \sin \theta_{W} \cos \theta_{W}}\left(1-4 \sin \theta_{W}^{2}\right), \\
\sum_{q=u, d} f_{V q}^{n} \lambda_{q, o}= & \frac{M_{Z}\left(\cos ^{2} \theta_{L}+\cos \theta_{R}^{2}\right)}{2 v} \times \frac{1}{M_{Z}^{2}} \\
& \times \frac{(-e)}{4 \sin \theta_{W} \cos \theta_{W}} .
\end{aligned}
$$

In the above formulas, $\theta_{W}$ is the weak-mixing angle and $\theta_{L, R}$ are the mixing angles defined in Eq. (17). Therefore, the total SI cross section can be written as

$$
\sigma_{N}^{\mathrm{SI}}=\sigma_{N, e}^{\mathrm{SI}}+\sigma_{N, o}^{\mathrm{SI}},
$$

where the vector SI cross section is given by (see $[11,44])$

$$
\begin{aligned}
\sigma_{N, o}^{\mathrm{SI}}= & \frac{G_{F}^{2} m_{N}^{2}}{4 \pi A^{2}}\left(\cos \theta_{L}^{2}+\cos \theta_{R}^{2}\right)^{2} \\
& \times\left[\left(1-4 \sin ^{2} \theta_{W}\right) Z-(A-Z)\right]^{2},
\end{aligned}
$$

and the scalar SI cross section is given by

$$
\sigma_{N, e}^{\mathrm{SI}} \approx \frac{m_{N}^{4} f_{N}^{2}}{\pi v^{2}}\left(\frac{g_{\chi_{1}^{0} \chi_{1}^{0} h_{1}}}{m_{h_{1}}^{2}}+\frac{g_{\chi_{1}^{0} \chi_{1}^{0} h_{2}}}{m_{h_{2}}^{2}}\right)^{2}
$$

with DM coupling to the Higgs fields written as

$g_{\chi_{1}^{0} \chi_{1}^{0} h_{k}}=\frac{-i}{\sqrt{2}} \sin \theta_{R}\left(h_{d} \cos \theta_{L} Z_{k 1}^{H}+h_{c} \sin \theta_{L} Z_{k 2}^{H}\right)$.

In the model presented in Ref. [11], which is a limiting case of our model and where the DM particle is mainly singlet, direct detection bounds imply that the mixing angles $\theta_{L, R}$ need to be very small. In that case, the only way to achieve the current value of the relic density of DM is via coannihilations, forcing the neutral fermions to be quasidegenerate, $M_{\Psi} \sim M_{N}$. In this work, however, that is not the case because of the presence of the new scalar $S$, which facilitates the depletion of DM during the early stages of the Universe. This allows us to obtain the correct relic density without coannihilations playing an important role, as we will show numerically in the next section.

Finally, the axial-vector interaction of DM with nucleons yields the SD scattering cross section, which has been probed by several experiments such as XENON1T [45] and LUX [46]. As we will see in Sec. IV C, the SD interactions provide less stringent restrictions on our scenario than the SI interactions.

\section{Numerical results}

In order to study the phenomenology of this model, we have performed a random scan of the parameter space, varying the free parameters as described in Table II. We implemented the model in SARAH [47-51], coupled to the SPheno $[52,53]$ routines. In order to obtain the DM relic

TABLE II. Scan range of the free parameters of our model. The remaining parameters are obtained from the ones in this table. In particular, $h_{b}^{\alpha 1}$ and $h_{b}^{\alpha 2}$ are fixed by Eq. (23), resulting in the range $10^{-8}<h_{b}^{\alpha 1, \alpha 2}<1$.

\begin{tabular}{lc}
\hline \hline Parameter & Range \\
\hline$M_{\Psi}(\mathrm{GeV})$ & $10^{2}-10^{4}$ \\
$m_{\sigma_{i}}(\mathrm{GeV})$ & $10^{3}-2 \times 10^{4}$ \\
$v_{S}(\mathrm{GeV})$ & $10^{2}-10^{5}$ \\
$\left|h_{c}\right|,\left|h_{d}\right|$ & $10^{-6}-3$ \\
$\lambda^{H \sigma_{i}}, \lambda^{S}, \lambda^{S \sigma_{i}}, \lambda_{S H}, \lambda^{\sigma_{i}}$ & $10^{-4}-3$ \\
$\left|h_{a}^{2 i, 3 i}\right|$ & $10^{-6}-1$ \\
\hline \hline
\end{tabular}



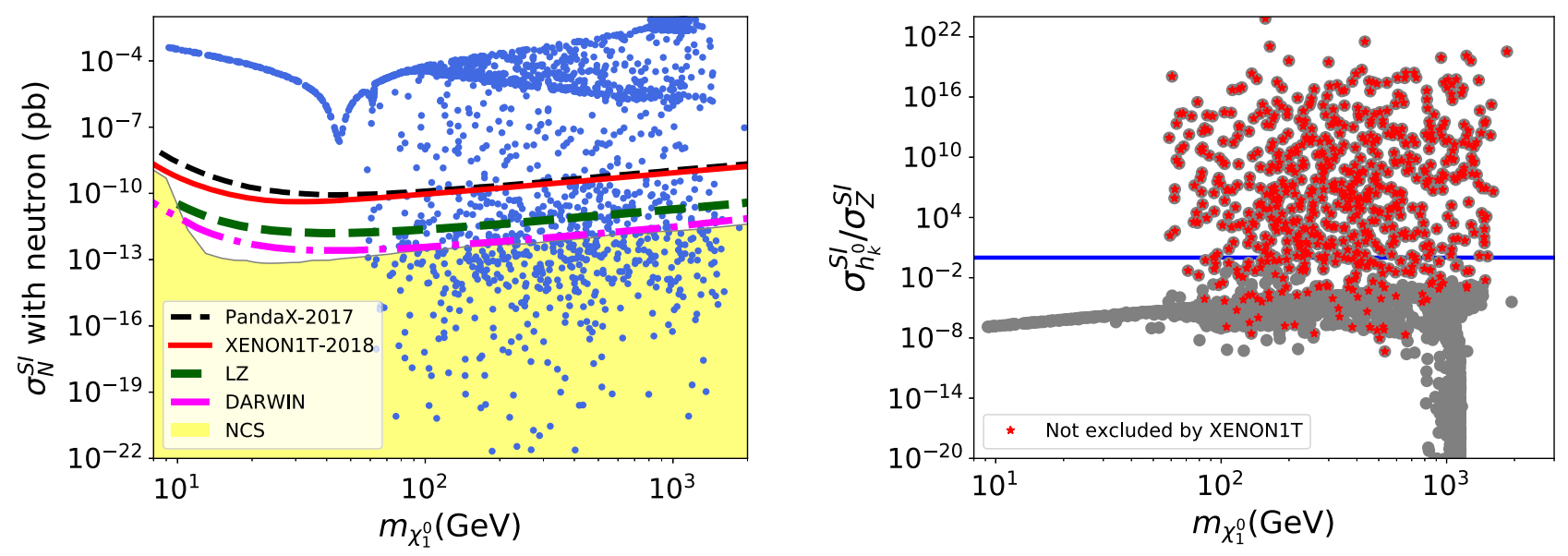

FIG. 4. Left: The SI cross section (blue dots) and the current experimental constraints from XENON1T [56], PandaX [57], and prospects from LZ [58] and DARWIN [59]. We also show the neutrino coherent scattering (NCS) [60,61]. Right: The grey dots show the ratio between the scalar and vector SI cross sections. The red stars are those models that are below the XENON1T limit.

density, we used MicrOMEGAs 4.2 .5 [54], which takes into account all the possible channels contributing to the relic density, mentioned in Sec. IVA, including special processes such as coannihilations and resonances [55]. We selected the models that fulfill the current value $\Omega_{\chi} h^{2}=$ $(0.120 \pm 0.001)$ to $3 \sigma[5]$ and, at the same time, reproduce the neutrino parameters described in Sec. III. For those points, we computed the SI DM-nucleus scattering cross section, shown in Eq. (33), and checked it against the current experimental bounds of XENON1T [56], PandaX [57], and prospect bounds of LZ [58] and DARWIN [59]. The results are shown in the left panel of Fig. 4. We analyzed the vector and scalar SI cross sections separately in order to discern the behavior of these two contributions to the total SI cross section and we found that the vector contribution dominates the region above the XENON1T limit. Therefore, it needs to be suppressed in order to escape the current bounds. The majority of models with a large vector SI cross section are excluded; these correspond to large mixing angles $\theta_{L, R}$, as seen from Eq. (34). Thus, the viable $\mathrm{DM}$ candidate needs to be mostly singlet in order to suppress the $Z$-portal and fulfill the current direct detection constraints. An analytic estimate tells us that this is achieved by requiring $\cos \theta_{L, R} \leq 0.1$. For illustrative purposes, in the right panel of Fig. 4, we show the ratio between the scalar and vector SI cross sections. The red stars correspond to the viable models that are not excluded by XENON1T. These models have a sizable scalar contribution $\left(\sigma_{N, e}^{\mathrm{SI}}=\sigma_{h_{k}^{0}}^{\mathrm{SI}}\right)$ and low vector cross section $\left(\sigma_{N, o}^{\mathrm{SI}}=\sigma_{Z}^{\mathrm{SI}}\right)$, except for some points that fall below the blue line which have a dominant vector cross section while escaping the direct detection (DD) bounds as analyzed in Ref. [11].

In order to complete this analysis, we show in the left panel of Fig. 5 the behavior of the WIMP-neutron spindependent $(\mathrm{SD})$ cross section for the points in the parameter space that yield the expected value of the relic abundance and reproduce the neutrino physics. We also show the IceCube [62] limits on the $W^{+} W^{-}$channel (black solid line) for DM annihilation at the sun, the limits from LUX [46] (yellow solid line), the current and most restricted limits from XENON1T [45] (green solid line), and the expected sensitivities of LZ [58] (red dashed line) and DARWIN [59] (magenta dotted line). As in the case of the SI cross section, we can see that DARWIN [59] could probe some region of the parameter space of this model. Evidently, the points that are below the neutrino floor could be confused with the neutrino scattering with nucleons and they would need a special analysis that is beyond the scope of this work.

Finally, in the right panel of Fig. 5, we show today's annihilation cross section times velocity, $\sigma v$, which allows us to look at indirect detection (ID) constraints. We used MicrOMEGAs 4.2 .5 to compute $\sigma v$ today for each point of the scan. Notice that these results show the expected suppression due to the $p$-wave nature of the DM annihilation. Therefore, the indirect DM detection prospects of this model are significantly low. For instance, the points with $m_{\chi_{1}^{0}} \lesssim 100 \mathrm{GeV}$ could have a large branching ratio of the annihilation channel $\chi_{1}^{0}{\overline{\chi_{1}}}^{0} \rightarrow b \bar{b}$, leading to DM annihilation into $b \bar{b}$ signals from dwarf galaxies (dSphs) [63]. However, as seen previously, those points are already excluded by DD. Combining the direct and indirect detection constraints, we conclude that all models with $m_{\chi_{1}^{0}} \lesssim 65 \mathrm{GeV}$ are excluded, except for the funnel region due to resonances with the $Z$ and the $h_{1}$ gauge bosons.

Following the analysis described above, we project the scanned points on the $M_{\Psi}-M_{N}$ plane and show it in Fig. 6. In the figure, the blue dots show the models that yield the correct value of the relic density and reproduce the neutrino parameters while the green-shaded region is excluded by DD experiments. The pink shade shows the 

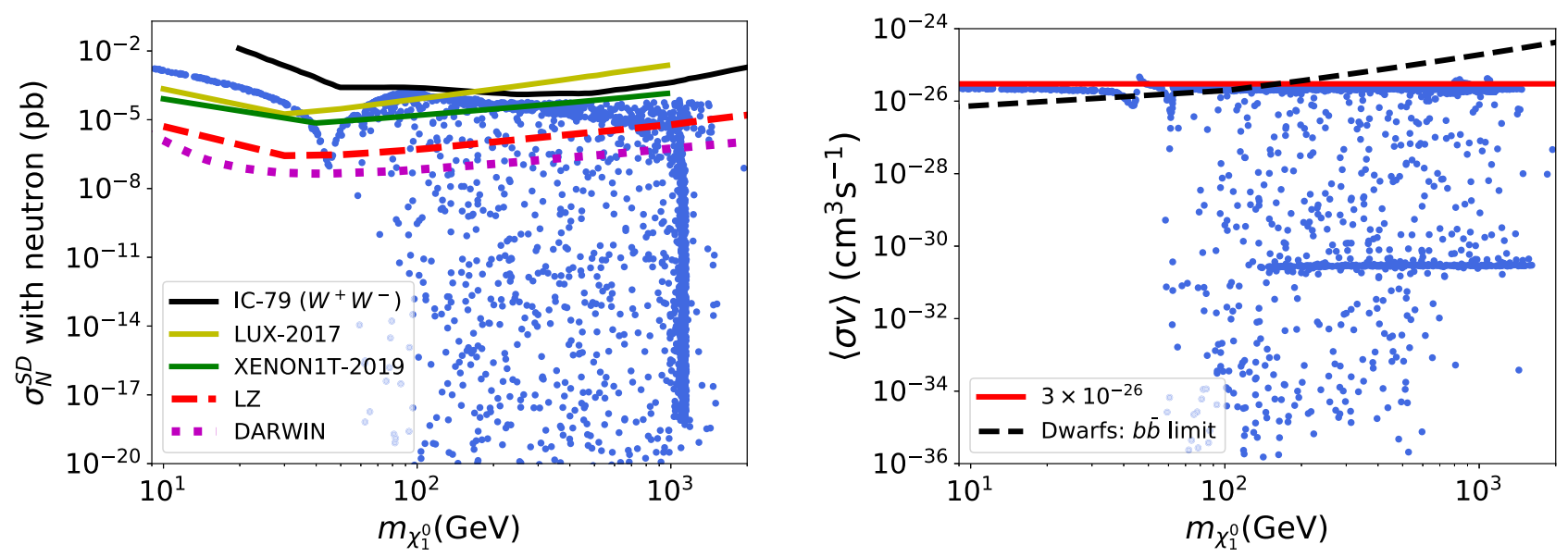

FIG. 5. Left: WIMP-neutron SD cross sections and the current experimental constraints from XENON1T [45], LUX [46], Ice-Cube [62], and prospects as LZ [58] and DARWIN [59]. Right: Annihilation cross section today. We also show the typical thermal value $\langle\sigma v\rangle \sim 3 \times 10^{-26} \mathrm{~cm}^{3} \mathrm{~s}^{-1}$ in the early Universe and the experimental limit for DM annihilation into $b \bar{b}$ in dwarf galaxies (dSphs) [63].

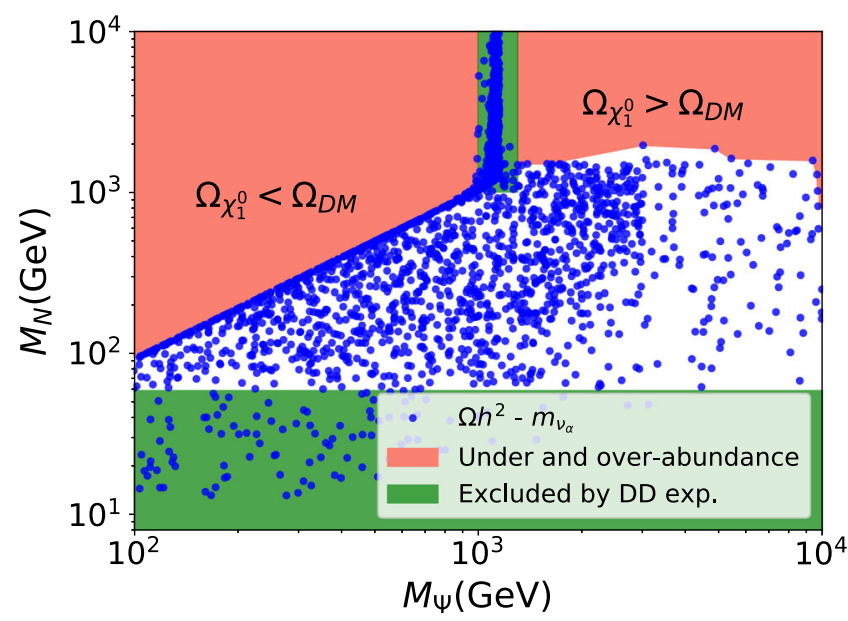

FIG. 6. $M_{\Psi}-M_{N}$ plane for the scan done in this work. The blue dots give the correct relic abundance and reproduce the neutrino parameters. The pink-shaded region corresponds to overabundance or underabundance of DM. The green-shaded region is excluded by DD experiments.

region where a larger, $\Omega_{\chi_{1}^{0}}>\Omega_{\mathrm{DM}}$, or smaller, $\Omega_{\chi_{1}^{0}}<\Omega_{\mathrm{DM}}$, relic density is obtained. Notice that, in our scenario, the region that leads to the correct abundance is much wider than in the Majorana fermion case $[16,17,19,20]$ and the original $\mathrm{SD}^{3} \mathrm{M}$ proposal [11], allowing the parameter $M_{N}$ up to $2 \mathrm{TeV}$ as shown in the figure.

In general, for models with $M_{\Psi}>M_{N}$, outside the region where coannihilations are relevant, the relic density is set through different channels in the early Universe. As argued in Sec. IVA, the main process is $\chi_{1}^{0}{\overline{\chi_{1}}}^{0} \rightarrow h_{2} h_{2}$. In that case, we have checked that the expression shown in Eq. (25) is in good agreement. Finally, in the coannihilation region $\left(M_{\Psi} \approx M_{N}\right)$, the main contributions to the relic density come from $\chi_{2}^{0} \chi^{+} \rightarrow f \bar{f}^{\prime}(\nu \bar{e}, u \bar{d}, \ldots)$ mediated by the $W^{ \pm}$boson, followed by $\chi_{2}^{0} \overline{\chi_{2}}{ }^{0} \rightarrow f \bar{f}$ and $\chi^{-} \chi^{+} \rightarrow f \bar{f}$. In this limit, processes involving the DM particle have a negligible contribution to the relic density because they are characterized by low Yukawa couplings as described in Ref. [11].

Finally, regarding collider searches, this scenario can be tested using the search for electroweak production of charginos $\chi^{ \pm}$decaying in final states with two leptons and missing transverse momentum in $p p$ collisions at the LHC [64]. Those analyses have been done in the context of simplified SUSY models and can be recast in this analysis. The observed limit rules out masses up to $120 \mathrm{GeV}$ for $\chi_{1}^{0}$, with $m_{\chi^{ \pm}} \lesssim 420 \mathrm{GeV}$. However, in that case, the $\chi^{ \pm}$are winolike particles with a production cross section that is larger than in this model, where $\chi^{ \pm}$are Higgsino-like particles $[S U(2)$ doublet]. With this in mind, we estimate that the low production rate decreases the values of $M_{N}$ that can be probed to $M_{N} \lesssim 100 \mathrm{GeV}$, which makes it inapplicable to our allowed region of parameter space. Nevertheless, a better analysis needs to be done in this direction and we leave it for future work.

\section{CONCLUSIONS}

After several decades of model building and experimental search, the nature of DM is still unknown. Among the many possible scenarios, a Dirac fermion is a viable candidate within the singlet-doublet scenario $\mathrm{SD}^{3} \mathrm{M}$ [11]. In this paper, we have minimally extended that model in order to generate Dirac neutrino masses via the radiative seesaw mechanism. We have scanned the parameter space requiring that the correct DM relic abundance and current neutrino data be reproduced while being compatible with direct detection experiments. We found a DM candidate that is a Dirac fermion resulting from a mixture of new singlet-doublet fields with mass $65 \mathrm{GeV} \lesssim m_{\chi_{1}^{0}} \lesssim 1.1 \mathrm{TeV}$. 
The inclusion of the new scalar $S$ opens a new portal, which, in association with the vector $Z$ portal, contributes to the SI cross section, widening the allowed parameter space while opening up the testing prospects in future direct detection experiments. Additionally, unlike in the original $\mathrm{SD}^{3} \mathrm{M}$ proposal, coannihilations do not play a central role in setting the relic abundance in our model. Regarding indirect detection, this framework does not provide clear prospective signatures since the annihilation cross section is $p$-wave suppressed.

\section{ACKNOWLEDGMENTS}

We are grateful to Oscar Zapata and Federico Von der Pahlen for enlightening discussions. D. R. is partially supported by COLCIENCIAS Grant No. 111577657253 and Sostenibilidad-UdeA. A. R. is supported by COLCIENCIAS through the ESTANCIAS POSTDOCTORALES program 2017, and by the American Physical Society (APS) International Research Travel Award Program. The research of W. T. is supported by the College of Arts and Sciences of Loyola University Chicago. A. R. is grateful to Loyola University Chicago (LUC) for the hospitality during the final stage of this work.

\section{APPENDIX: LAGRANGIAN IN TERMS OF WEYL SPINORS}

The Lagrangian in Eq. (1) can be written in terms of chiral spinors as follows:

$$
\begin{aligned}
\mathcal{L} \supset-M_{\Psi} \bar{\Psi} \Psi & =-M_{\Psi}\left(\overline{\Psi^{0}}, \overline{\Psi^{-}}\right)\left(\begin{array}{c}
\Psi^{0} \\
\Psi^{-}
\end{array}\right) \\
& =-M_{\Psi}\left(\overline{\Psi^{0}} \Psi^{0}+\overline{\Psi^{-} \Psi^{-}}\right)=-M_{\Psi}\left(\left(\Psi^{0}\right)^{\dagger} \gamma_{0} \Psi^{0}+\left(\Psi^{-}\right)^{\dagger} \gamma_{0} \Psi^{-}\right) \\
& =-M_{\Psi}\left[\left(\Psi_{R}^{0}, \Psi_{L}^{0}\right)^{\dagger}\left(\begin{array}{cc}
0 & 1 \\
1 & 0
\end{array}\right)\left(\begin{array}{c}
\Psi_{R}^{0} \\
\Psi_{L}^{0}
\end{array}\right)+\left(\Psi_{R}^{-}, \Psi_{L}^{-}\right)^{\dagger}\left(\begin{array}{cc}
0 & 1 \\
1 & 0
\end{array}\right)\left(\begin{array}{c}
\Psi_{R}^{-} \\
\Psi_{L}^{-}
\end{array}\right)\right] \\
& =-M_{\Psi}\left[\left(\Psi_{L}^{0}\right)^{\dagger} \Psi_{R}^{0}+\left(\Psi_{L}^{-}\right)^{\dagger} \Psi_{R}^{-}+\text {H.c. }\right] \\
& =-M_{\Psi}\left[\left(-\left(\Psi_{R}^{-}\right)^{\dagger},\left(\Psi_{R}^{0}\right)^{\dagger}\right)\left(\begin{array}{cc}
0 & -1 \\
1 & 0
\end{array}\right)\left(\begin{array}{c}
\Psi_{L}^{0} \\
\Psi_{L}^{-}
\end{array}\right)+\text {H.c. }\right]=-M_{\Psi}\left[\left(\widetilde{\left.\Psi_{R}\right)} \cdot \Psi_{L}+\text { H.c. }\right],\right.
\end{aligned}
$$

where the dot product represents the $i \sigma_{2}$ matrix and $\widetilde{\left(\Psi_{R}\right)}=\left(-\left(\Psi_{R}^{-}\right)^{\dagger},\left(\Psi_{R}^{0}\right)^{\dagger}\right)^{T}, \Psi_{L}=\left(\Psi_{L}^{0}, \Psi_{L}^{-}\right)^{T}$ are two chiral doublets of $S U(2)$ with opposite hypercharge. In the same way,

$$
\begin{aligned}
& \mathcal{L} \supset h_{d} \bar{\Psi} \tilde{H} \psi_{R}+\text { H.c. }=h_{d}\left(\overline{\Psi^{0}}, \overline{\Psi^{-}}\right)\left(\begin{array}{c}
\left(H^{0}\right)^{*} \\
-H^{-}
\end{array}\right) \psi_{R}+\text { H.c. }=h_{d}\left[\left(\Psi_{L}^{0}\right)^{\dagger}\left(H^{0}\right)^{*} \psi_{R}-\left(\Psi_{L}^{-}\right)^{\dagger} H^{-} \psi_{R}+\text { H.c. }\right] \\
&=h_{d}\left[\left(\psi_{R}\right)^{\dagger} H^{0} \Psi_{L}^{0}-\left(\psi_{R}\right)^{\dagger} H^{+} \Psi_{L}^{-}+\text {H.c. }\right]=h_{d}\left(\psi_{R}\right)^{\dagger}\left(H^{+}, H^{0}\right)\left(\begin{array}{cc}
0 & -1 \\
1 & 0
\end{array}\right)\left(\begin{array}{c}
\Psi_{L}^{0} \\
\Psi_{L}^{-}
\end{array}\right)+\text {H.c. } \\
&=h_{d}\left(\psi_{R}\right)^{\dagger} H \cdot \Psi_{L}+\text { H.c. } \\
& \mathcal{L} \supset h_{a}^{\beta i} \bar{L}_{\beta} \Psi \sigma_{i}+\text { H.c. }=h_{a}^{\beta i}\left(\left(\nu_{L}\right)_{\beta}^{\dagger} \Psi_{R}^{0}+\left(e_{L}\right)_{\beta}^{\dagger} \Psi_{R}^{-}\right) \sigma_{i}+\text { H.c. }=h_{a}^{\beta i}\left(\left(\Psi_{R}^{0}\right)^{\dagger}\left(\nu_{L}\right)_{\beta}+\left(\Psi_{R}^{-}\right)^{\dagger}\left(e_{L}\right)_{\beta}\right) \sigma_{i}+\text { H.c. } \\
&=h_{a}^{\beta i}\left(-\left(\Psi_{R}^{-}\right)^{\dagger},\left(\Psi_{R}^{0}\right)^{\dagger}\right)\left(\begin{array}{cc}
0 & -1 \\
1 & 0
\end{array}\right)\left(\begin{array}{c}
\left(\nu_{L}\right)_{\beta} \\
\left(e_{L}\right)_{\beta}
\end{array}\right) \sigma_{i}+\text { H.c. }=h_{a}^{\beta i}\left(\widetilde{\Psi_{R}}\right) \cdot L_{\beta} \sigma_{i}+\text { H.c. }
\end{aligned}
$$

$\mathcal{L} \supset h_{b}^{\alpha i} \overline{\psi_{L}} \nu_{R \alpha} \sigma_{i}+h_{c} \overline{\psi_{R}} \psi_{L} S+$ H.c. $=h_{b}^{\alpha i} \bar{\psi} P_{R} \nu_{\alpha} \sigma_{i}+h_{c} \bar{\psi} P_{L} \psi S+$ H.c.

$$
\begin{aligned}
& =h_{b}^{\alpha i}\left(\psi_{R}, \psi_{L}\right)^{\dagger}\left(\begin{array}{cc}
0 & 1 \\
1 & 0
\end{array}\right)\left(\begin{array}{c}
\nu_{R \alpha} \\
0
\end{array}\right) \sigma_{i}+h_{c}\left(\psi_{R}, \psi_{L}\right)^{\dagger}\left(\begin{array}{ll}
0 & 1 \\
1 & 0
\end{array}\right)\left(\begin{array}{c}
0 \\
\psi_{L}
\end{array}\right) S+\text { H.c. } \\
& =h_{b}^{\alpha i}\left(\psi_{L}\right)^{\dagger} \nu_{R \alpha} \sigma_{i}+h_{c}\left(\psi_{R}\right)^{\dagger} \psi_{L} S+\text { H.c. }
\end{aligned}
$$

Therefore, replacing Eqs. (A1), (A2), (A3), and (A4) in Eq. (1), we obtain

$$
\begin{aligned}
\mathcal{L} \supset & -M_{\Psi}\left[\widetilde{\left(\widetilde{\Psi_{R}}\right)} \cdot \Psi_{L}+\text { H.c. }\right]-V\left(H, \sigma_{i}, S\right) \\
& +\left[h_{a}^{\beta i}\left(\widetilde{\left.\Psi_{R}\right)} \cdot L_{\beta} \sigma_{i}+h_{b}^{\alpha i}\left(\psi_{L}\right)^{\dagger} \nu_{R \alpha} \sigma_{i}+h_{c}\left(\psi_{R}\right)^{\dagger} \psi_{L} S+h_{d}\left(\psi_{R}\right)^{\dagger} H \cdot \Psi_{L}+\text { H.c. }\right] .\right.
\end{aligned}
$$


[1] F. Zwicky, On the masses of nebulae and of clusters of nebulae, Astrophys. J. 86, 217 (1937).

[2] P. Gorenstein and W. Tucker, Astronomical signatures of dark matter, Adv. High Energy Phys. 2014, 1 (2014).

[3] V. C. Rubin and W. K. Ford, Jr., Rotation of the Andromeda nebula from a spectroscopic survey of emission regions, Astrophys. J. 159, 379 (1970).

[4] V. C. Rubin, N. Thonnard, and W. K. Ford, Jr., Rotational properties of $21 \mathrm{SC}$ galaxies with a large range of luminosities and radii, from NGC $4605 / R=4 \mathrm{kpc} /$ to UGC $2885 / R=122 \mathrm{kpc} /$, Astrophys. J. 238, 471 (1980).

[5] N. Aghanim et al. (Planck Collaboration), Planck 2018 results. VI. Cosmological parameters, arXiv:1807.06209.

[6] D. Clowe, M. Bradac, A. H. Gonzalez, M. Markevitch, S. W. Randall, C. Jones, and D. Zaritsky, A direct empirical proof of the existence of dark matter, Astrophys. J. 648, L109 (2006).

[7] A. Refregier, Weak gravitational lensing by large scale structure, Annu. Rev. Astron. Astrophys. 41, 645 (2003).

[8] J. A. Tyson, G. P. Kochanski, and I. P. Dell'Antonio, Detailed mass map of CL0024+1654 from strong lensing, Astrophys. J. 498, L107 (1998).

[9] C. S. Frenk and S. D. M. White, Dark matter and cosmic structure, Ann. Phys. (Amsterdam) 524, 507 (2012).

[10] P. F. de Salas, D. V. Forero, C. A. Ternes, M. Tortola, and J. W. F. Valle, Status of neutrino oscillations 2018: $3 \sigma$ hint for normal mass ordering and improved $C P$ sensitivity, Phys. Lett. B 782, 633 (2018).

[11] C. E. Yaguna, Singlet-doublet Dirac dark matter, Phys. Rev. D 92, 115002 (2015).

[12] N. Arkani-Hamed, S. Dimopoulos, and S. Kachru, Predictive landscapes and new physics at a TeV, arXiv:hep-th/ 0501082.

[13] R. Mahbubani and L. Senatore, The minimal model for dark matter and unification, Phys. Rev. D 73, 043510 (2006).

[14] F. D'Eramo, Dark matter, and Higgs boson physics, Phys. Rev. D 76, 083522 (2007).

[15] R. Enberg, P. Fox, L. Hall, A. Papaioannou, and M. Papucci, LHC and dark matter signals of improved naturalness, J. High Energy Phys. 11 (2007) 014.

[16] T. Cohen, J. Kearney, A. Pierce, and D. Tucker-Smith, Singletdoublet dark matter, Phys. Rev. D 85, 075003 (2012).

[17] C. Cheung and D. Sanford, Simplified models of mixed dark matter, J. Cosmol. Astropart. Phys. 02 (2014) 011.

[18] T. Abe, R. Kitano, and R. Sato, Discrimination of dark matter models in future experiments, Phys. Rev. D 91, 095004 (2015).

[19] D. Restrepo, A. Rivera, M. Sánchez-Peláez, O. Zapata, and W. Tangarife, Radiative neutrino masses in the singletdoublet fermion dark matter model with scalar singlets, Phys. Rev. D 92, 013005 (2015).

[20] L. Calibbi, A. Mariotti, and P. Tziveloglou, Singlet-doublet model: Dark matter searches and LHC constraints, J. High Energy Phys. 10 (2015) 116.

[21] S. Horiuchi, O. Macias, D. Restrepo, A. Rivera, O. Zapata, and H. Silverwood, The Fermi-LAT gamma-ray excess at the galactic center in the singlet-doublet fermion dark matter model, J. Cosmol. Astropart. Phys. 03 (2016) 048.
[22] S. Bhattacharya, P. Ghosh, and N. Sahu, Multipartite dark matter with scalars, fermions and signatures at LHC, J. High Energy Phys. 02 (2019) 059.

[23] S. Bhattacharya, P. Ghosh, N. Sahoo, and N. Sahu, A minireview on vector-like leptonic dark matter, neutrino mass and collider signatures, Front. Phys. 7, 80 (2019).

[24] B. J. Mount et al., LUX-ZEPLIN (LZ) Technical Design Report, arXiv:1703.09144.

[25] E. Ma, Verifiable radiative seesaw mechanism of neutrino mass and dark matter, Phys. Rev. D 73, 077301 (2006).

[26] S. Centelles Chuliá, E. Ma, R. Srivastava, and J. W. F. Valle, Dirac neutrinos and dark matter stability from lepton quarticity, Phys. Lett. B 767, 209 (2017).

[27] S. Centelles Chuliá, R. Srivastava, and J. W. F. Valle, Seesaw roadmap to neutrino mass and dark matter, Phys. Lett. B 781, 122 (2018).

[28] J. Calle, D. Restrepo, C. E. Yaguna, and O. Zapata, Minimal radiative Dirac neutrino mass models, Phys. Rev. D 99, 075008 (2019).

[29] K. Kannike, Vacuum stability conditions from copositivity criteria, Eur. Phys. J. C 72, 2093 (2012).

[30] A. Falkowski, C. Gross, and O. Lebedev, A second Higgs from the Higgs portal, J. High Energy Phys. 05 (2015) 057.

[31] M. Aaboud et al. (ATLAS Collaboration), Measurements of Higgs boson properties in the diphoton decay channel with $36 \mathrm{fb}^{-1}$ of $p p$ collision data at $\sqrt{s}=13 \mathrm{TeV}$ with the ATLAS detector, Phys. Rev. D 98, 052005 (2018).

[32] The ATLAS collaboration, Measurements of Higgs boson properties in the diphoton decay channel using $80 \mathrm{fb}^{-1}$ of $p p$ collision data at $\sqrt{s}=13 \mathrm{TeV}$ with the ATLAS detector, Report No. ATLAS-CONF-2018-028, 2018.

[33] A. M. Sirunyan et al. (CMS Collaboration), Measurements of Higgs boson properties in the diphoton decay channel in proton-proton collisions at $\sqrt{s}=13 \mathrm{TeV}$, J. High Energy Phys. 11 (2018) 185.

[34] V. Khachatryan et al. (CMS Collaboration), Search for a Higgs boson in the mass range from 145 to $1000 \mathrm{GeV}$ decaying to a pair of $\mathrm{W}$ or $\mathrm{Z}$ bosons, J. High Energy Phys. 10 (2015) 144.

[35] D. Restrepo, O. Zapata, and C. E. Yaguna, Models with radiative neutrino masses and viable dark matter candidates, J. High Energy Phys. 11 (2013) 011.

[36] C.-Y. Yao and G.-J. Ding, Systematic analysis of Dirac neutrino masses from a dimension five operator, Phys. Rev. D 97, 095042 (2018).

[37] S. Kanemura, T. Nabeshima, and H. Sugiyama, Neutrino masses from loop-induced Dirac Yukawa couplings, Phys. Lett. B 703, 66 (2011).

[38] Z. Maki, M. Nakagawa, and S. Sakata, Remarks on the unified model of elementary particles, Prog. Theor. Phys. 28, 870 (1962).

[39] E. W. Kolb and M. S. Turner, The early universe, Front. Phys. 69, 1 (1990).

[40] M. Srednicki, R. Watkins, and K. A. Olive, Calculations of relic densities in the early universe, Nucl. Phys. B310, 693 (1988).

[41] G. Belanger, F. Boudjema, A. Pukhov, and A. Semenov, Dark matter direct detection rate in a generic model with micrOMEGAs 2.2, Comput. Phys. Commun. 180, 747 (2009). 
[42] J. M. Alarcon, J. Martin Camalich, and J. A. Oller, The chiral representation of the $\pi N$ scattering amplitude and the pion-nucleon sigma term, Phys. Rev. D 85, 051503 (2012).

[43] J. M. Alarcon, L. S. Geng, J. Martin Camalich, and J. A. Oller, The strangeness content of the nucleon from effective field theory and phenomenology, Phys. Lett. B 730, 342 (2014).

[44] J. D. Lewin and P.F. Smith, Review of mathematics, numerical factors, and corrections for dark matter experiments based on elastic nuclear recoil, Astropart. Phys. 6, 87 (1996).

[45] E. Aprile et al. (XENON Collaboration), Constraining the Spin-Dependent WIMP-Nucleon Cross Sections with XENON1T, Phys. Rev. Lett. 122, 141301 (2019).

[46] D. S. Akerib et al. (LUX Collaboration), Limits on SpinDependent WIMP-Nucleon Cross Section Obtained from the Complete LUX Exposure, Phys. Rev. Lett. 118, 251302 (2017).

[47] F. Staub, SARAH, arXiv:0806.0538.

[48] F. Staub, From superpotential to model files for FeynArts and CalcHep/CompHep, Comput. Phys. Commun. 181, 1077 (2010).

[49] F. Staub, Automatic calculation of supersymmetric renormalization group equations and self energies, Comput. Phys. Commun. 182, 808 (2011).

[50] F. Staub, SARAH 3.2: Dirac gauginos, UFO output, and more, Comput. Phys. Commun. 184, 1792 (2013).

[51] F. Staub, SARAH 4: A tool for (not only SUSY) model builders, Comput. Phys. Commun. 185, 1773 (2014).

[52] W. Porod, SPheno, a program for calculating supersymmetric spectra, SUSY particle decays and SUSY particle production at e+ e- colliders, Comput. Phys. Commun. 153, 275 (2003).

[53] W. Porod and F. Staub, SPheno 3.1: Extensions including flavour, $C P$-phases and models beyond the MSSM, Comput. Phys. Commun. 183, 2458 (2012).

[54] G. Belanger, F. Boudjema, A. Pukhov, and A. Semenov, MicrOMEGAs 2.0: A program to calculate the relic density of dark matter in a generic model, Comput. Phys. Commun. 176, 367 (2007).

[55] K. Griest and D. Seckel, Three exceptions in the calculation of relic abundances, Phys. Rev. D 43, 3191 (1991).

[56] E. Aprile et al. (XENON Collaboration), Dark Matter Search Results from a One Tonne $\times$ Year Exposure of XENON1T, Phys. Rev. Lett. 121, 111302 (2018).

[57] X. Cui et al. (PandaX-II Collaboration), Dark Matter Results from 54-Ton-Day Exposure of PandaX-II Experiment, Phys. Rev. Lett. 119, 181302 (2017).

[58] D. S. Akerib et al. (LUX-ZEPLIN Collaboration), Projected WIMP sensitivity of the LUX-ZEPLIN (LZ) dark matter experiment, arXiv:1802.06039.

[59] J. Aalbers et al. (DARWIN Collaboration), DARWIN: Towards the ultimate dark matter detector, J. Cosmol. Astropart. Phys. 11 (2016) 017.

[60] P. Cushman et al., Proceedings, 2013 Community Summer Study on the Future of U.S. Particle Physics: Snowmass on the Mississippi (CSS2013), edited by N. A. Graf, M. E. Peskin, and J. L. Rosner (2013), http://www.slac.stanford .edu/econf/C1307292.

[61] J. Billard, L. Strigari, and E. Figueroa-Feliciano, Implication of neutrino backgrounds on the reach of next generation dark matter direct detection experiments, Phys. Rev. D 89, 023524 (2014).

[62] M. G. Aartsen, R. Abbasi, Y. Abdou, M. Ackermann, J. Adams, J. A. Aguilar, M. Ahlers, D. Altmann, J. Auffenberg, X. Bai et al., Search for Dark Matter Annihilations in the Sun with the 79-String IceCube Detector, Phys. Rev. Lett. 110, 131302 (2013).

[63] M. Ackermann et al. (Fermi-LAT Collaboration), Searching for Dark Matter Annihilation from Milky Way Dwarf Spheroidal Galaxies with Six Years of Fermi-LAT Data, Phys. Rev. Lett. 115, 231301 (2015).

[64] The ATLAS collaboration, Search for electroweak production of charginos and sleptons decaying in final states with two leptons and missing transverse momentum in $\sqrt{\mathrm{s}}=$ $13 \mathrm{TeV} p p$ collisions using the ATLAS detector, Report No. ATLAS-CONF-2019-008, 2019. 